\title{
Soumission: una fenomenología de la academia ${ }^{1}$
}

\section{(Soumission: A Phenomenology of Academia)}

\section{Roy Alfaro Vargas 2}

Universidad de Costa Rica

\begin{abstract}
RESUMEN
Se analiza la novela Soumission de Michel Houellebecq, en relación con su personaje central François, para describir la dialéctica entre el hedonismo neoliberal y fenomenológico, y lo económico, en tanto crítica de la academia, en el contexto de la actual crisis del capitalismo. Asimismo, el análisis permite la elaboración de una poética realista-dialéctica relacionada con el extrañamiento cognitivo de la ciencia ficción y con el realismo de Balzac, Zola y Flaubert, para contrarrestar la política de lo imposible desarrollada en las narrativas innaturales y el novum tecnocrático.
\end{abstract}

\begin{abstract}
Michel Houllebecq's Soumission is analyzed in relation to its central character François, to describe the dialectics between neoliberal and phenomenological hedonism and economic issues, as a critique of academia, in the context of the current crisis of capitalism. The analysis also allows the elaboration of a realist-dialectical poetics regarding the cognitive estrangement of science fiction and the realism of Balzac, Zola, and Flaubert, to counteract the politics of the impossible developed in unnatural narratives and the technocratic novum.
\end{abstract}

1 Recibido: 3 de mayo de 2018; aceptado: 17 de setiembre de 2018. El autor le agradece a Diego Chacón Porras su colaboración.

2 Correo electrónico: royalfarov@yandex.com.

LETRAS 64 (2018), ISSN 1409-424X; EISSN 2215-4094 
Palabras clave: literatura francesa, novela francesa contemporánea, novela experimental, Michel Houellebecq

Keywords: French literature, contemporary French novel, experimental novel, Michel Houellebecq

\section{Introducción}

En la literatura francesa contemporánea, Michel Houellebecq, con su novela Soumission ${ }^{3}$, presenta un proceso de islamización en la Francia de 2022, en tono desafiante e irónico, pleno de crítica política y misoginia, en un ambiente estético realista. Houellebecq es poco conocido en nuestro contexto pero, a la vez, es un escritor que ofrece, para el desarrollo de la literatura costarricense y en general, algunas líneas poiéticas que permitirían una mejora de la producción literaria de nuestro país y más allá de nuestras fronteras.

En tal marco, se plantea la siguiente tesis: Michel Houellebecq, en Soumission, establece una dialéctica entre el hedonismo neoliberal ligado a la fenomenología y la materialidad socioeconómica, mediante el personaje central (François), como un medio de crítica tanto de la política, como de la academia, y como fundamento de una poética realista-dialéctica. Para llevar a término la tesis, es preciso, en primer lugar, definir ciertas características de la poética houellebecquiana; segundo, establecer la relación entre François y el hedonismo neoliberal de la fenomenología actual; tercero, determinar las inconsistencias del hedonismo neoliberal de François, en su dialéctica con lo socioeconómico, en tanto crítica política y de lo académico. Finalmente, se debe definir la poética realisto-dialéctica, que se deriva de la lectura de Soumission.

3 Michel Houellebecq, Soumission (París: Flammarion, 2015). 


\section{La poética houellebecquiana}

Michel Houellebecq (Michel Thomas su nombre real) nació el 26 de febrero de 1956, en Réunion. Estudió cine, agronomía y tecnologías de la información. Entre sus libros de poesía están: $L a$ poursuite du bonheur (1992) y Le sens du combat (1996). Su primera novela fue Extension du domaine de la lutte (1994), junto a la cual están Les particles élémentaires (1998), Plateforme (2001), La carte et le territoire (2010) (Premio Prix Goncourt) ${ }^{4}$.Además, La possibilité d'une île (2005) ha sido llevada al cine en el $2008^{5}$.

En términos estéticos, la novela houellebecquiana se asocia al realismo de Balzac, Zola y Flaubert ${ }^{6}$, de donde «hereda un cierto gusto por la controversia» ${ }^{7}$ (mi traducción), que también inserta a Houellebecq entre los escritores «que operan un "retorno a lo real" ${ }^{8}$ (mi traducción), y en cuyo caso la literatura sirve «como instrumento de interpretación de la contemporaneidad $»^{9}$ (mi traducción):

La novela houellebecquiana marca el retorno de la novela social de principios del siglo XIX. El realismo de sus novelas, utilizado con la precisión quirúrgica de la escritura que se quiere falsamente neutra y objetiva, es uno de los elementos estéticos más asombrosos. En la línea de la novela naturalista, Houellebecq describe el universo

4 John Flower, Historical Dictionary of French Literature (Lanham, Toronto y Plymouth: The Scarecrow Press, 2013) 246-248.

5 La possibilité d'île. Dir. Michel Houellebecq. Con Benoît Magimel, Ramata Koite, Patrick Bauchau y otros. Westdeutscher Rundfunk (WDR), Canal+, Filmstiftung North Rhine Westphalia, Mandarin Films, Morena Films, Black Forest Films, Motion Investment Group, Cofinova 3, Pictorion Pictures GmbH, Cofinova 4, WAT Productions, arte France Cinéma, Lagardère, Studio 37, Michel Houellebecq, Instituto de la Cinematografía y de las Artes Audiovisuales (ICAA), Soficinéma 3, 2008.

6 Marc Smeets, «Michel Houellebecq: un homme, une (sou)mission», Relief 9, 2 (2015): 99-111. DOI:10.18352/ relief.919

7 Smeets, 99.

8 Raphaël Baroni, «Comment débusquer la voix d'un auteur dans sa fiction? Une étude de quelques provocations de Michel Houellebecq», Arborescence 6 (2016): 72-93. DOI: 10.7202/1037505ar.

9 Silvia Annavini, «Soumission. Michel Houellebecq e la conversion alla letteratura», Between III, 5 (2015): 1-5. DOI: http://dx.doi.org/10.13125/2039-6597/2034. 
cotidiano del trabajo occidental, minuciosamente autopsiado en toda su imbecilidad y su alienación triunfantes ${ }^{10}$ (mi traducción).

Así, su novela se orienta a la cotidianidad de un capitalismo en crisis terminal, alrededor de los imperativos neoliberales, con una estética realisto-naturalista crítica. En este marco, son novelas que se levantan sobre «el trabajo de investigación y de observación como algo indispensable, previo a toda creación novelesca, inventando una forma única, a saber, la novela experimental» ${ }^{11}$ (mi traducción). La novela experimental de Houellebecq, con elementos como la ironía, la evocación de la miseria, la crítica de Occidente ${ }^{12}$, así como su reflexión sobre lo económico-social en las sociedades post-industriales ${ }^{13}$ y su apropiación tanto de lo pornográfico como del discurso científico ${ }^{14}$, en un universo «deliberadamente en las mismas coordenadas que la globalización» ${ }^{15}$ (mi traducción); es «como una útil y saludable provocación, como un texto programado para suscitar una recepción confusa, como un arma de combate y de resistencia» ${ }^{16}$ (mi traducción).

La novela experimental houellebecquiana se liga a la influencia de Arthur Schopenhauer, lo cual la marca con una tensión entre sufrimiento y deseo ${ }^{17}$, «donde el deseo es muerte» ${ }^{18}$ (mi traducción) y el sufrimiento se presenta como desesperanza relacional, la cual «devela el nihilismo actuante detrás del hedonismo de hoy» ${ }^{19}$ (mi traducción),

10 Sandrine Rabosseau, «Houellebecq ou le renouveau du roman expérimental», Michel Houellebecq sous la loupe (Amsterdam y New York: Radopi, 2007) 44.

11 Rabosseau, 43.

12 Rabosseau, 45.

13 Ruth Amar, «Michel Houellebecq: The Era of Emptiness», Journalism and Mass Communication 6, 3 (2016): 158-165. DOI:10.17265/2160-6579/2016.03.004.

14 Simon St-Onge, «De l'esthétique houellebecquienne», Michel Houellebecq sous la loupe (Amsterdam y New York: Radopi, 2007) 70.

15 Daniel Laforest, «Mondialisation, espace et séparation chez Michel Houellebecq», Michel Houellebecq sous la loupe (Amsterdam y New York: Radopi, 2007) 265.

16 Rabosseau, 45.

17 Floriane Place-Verghnes, «Houellebecq/Schopenhauer: Souffrance et désir gigognes», Michel Houellebecq sous la loupe (Amsterdam y New York: Radopi, 2007) 123-132.

18 Claire Arènes y Jacques Arènes, «Michel Houellebecq: prophète des temps finissants», Études 6, 404 (2006): 796-803.

19 Arènes, 801. 
«haciendo estallar la ilusión postmoderna de una relación armoniosa en la alteridad $\gg^{20}$ (mi traducción). De todo esto, se derivan dos hechos: primero, la descuartización de los personajes houellebecquianos, quienes condensan el aspecto mimético y el pensamiento del escritor, a la vez que se (re)presentan como constructos autónomos con voz propia $^{21} ; \mathrm{y}$ en segundo lugar, los personajes se transforman en el ámbito profesional y privado, marcados por la bestialidad y la frustración ${ }^{22}$, reproduciendo un tema central de las novelas houellebecquianas, o sea, las relaciones amorosas y sociales se asientan sobre el modelo de las relaciones económicas ${ }^{23}$.

Por otra parte, la obra de Houellebecq recibe la influencia de la ciencia ficción, lo cual se sustenta no solo en algunas de sus textos propiamente escritos dentro de los parámetros de este género (por ejemplo: La possibilité d'une île ${ }^{24}$ ), sino también en la estructuración del relato mediante una reelaboración del mecanismo suviniano del extrañamiento cognitivo ${ }^{25}$; en términos houellebecquianos, se representa la acción en una perspectiva futurista, con el fin «de analizar esta humanidad desde una perspectiva crítica» ${ }^{26}$ (mi traducción). Es decir, se desplaza la situación del hoy (dentro del ámbito estético del realismo) hacia el futuro, para permitirle al lector desprenderse del vínculo emocional con la situación actual, buscando que el lector aprehenda cognitivamente su situación socioeconómica y política, en confrontación con el texto literario y con ello generar conciencia política en el lector mismo. Con este manejo de la temporalidad (que rompe con el realismo decimonónico de Zola, Balzac, etc.), la literatura se define entonces «como un instrumento de interpretación de

20 Laforest, 266.

21 Baroni, 78.

22 Rabosseau, 49.

23 Olivier Bessard-Banquy, «Le degré zéro de l'écriture selon Houellebecq», Michel Houellebecq sous la loupe (Amsterdam y New York: Radopi, 2007) 357.

24 Michel Houellebecq, La possibilité d'une île (París: Fayard, 2005).

25 Darko Suvin, Metamorphoses of Science Fiction. On the Poetics and History of a Literary Genre (New Haven y Londres: Yale University Press, 1979).

26 Annavini, 5. 
la contemporaneidad $»^{27}$ (mi traducción), al igual que como proyecto político. (Aquí está la base de lo que más adelante llamaremos realismo dialéctico).

\section{François, neoliberalismo y hedonismo}

En Soumission, François representa la intelligentsia francesa, la europea, la primermundista y la global. Sin embargo, también es en el elemento metonímico donde converge la noción de europeidad, como factor identitario, en el contexto del auge neoliberal y de la globalización; así como el hedonismo fenomenológico y fenomenologizante que funda el exacerbado individualismo narcisista y egoísta de nuestra época: «Houellebecq individua la academia como alegoría del fallo intelectual que envuelve al sistema occidental entero $»^{28}(\mathrm{mi}$ traducción), pero también al sistema capitalista como totalidad.

Asimismo, François representa, como corporeidad, el choque entre las promesas discursivas post-postmodernas del hedonismo de sesgo fenomenológico y la materialidad ligada, en Soumission, a lo económico. Por ende, François requiere ser interpretado tanto en términos sociológicos, como filosóficos, con el fin de hallar la síntesis antropológica que sostiene a este personaje.

El eje que justifica la existencia de François es el placer, específicamente el placer sexual:

L'amour chez l'homme n'est rien d'autre que la reconnaissance pour le plaisir donné, et jamais personne ne m'avait donné autant de plaisir que Myriam. Elle pouvait contracter sa chatte à volonté (tantôt doucement, par lentes pressions irrésistibles, tantôt par petites secousses vives et mutines) ; elle tortillait son petit cul avec une grâce infinie avant de me l'offrir. Quant à ses fellations, je n'avais jamais rien connu de semblable, elle abordait chaque fellation comme si c'était la première, et que ce devait être la dernière de sa vie.

27 Annavini, 1.

28 Annavini, 7. 
Chacune de ses fellations aurait suffi à justifier la vie d'un homme 29 . [El amor en el hombre no es más que el reconocimiento del placer dado y nunca nadie me había dado tanto placer como Myriam. Ella podía contraer su pepa a voluntad (a veces dulcemente por medio de lentas e irresistibles presiones, a veces por pequeñas sacudidas vivas y divertidas); ella retorcía su pequeño culo con una gracia infinita antes de ofrecérmelo. En cuanto a sus felaciones, yo no había conocido nunca nada semejante, ella abordaba cada felación como si fuese la primera y como si esta fuera la última de su vida. Cada una de sus felaciones habría bastado para justificar la vida de un hombre.]

El placer de François es el evento (su Ereignis) de su vivencia (Erlebnis). Es un académico cuyo único interés es el placer sexual, el cual da sentido a su vida por encima de su presunta intelectualidad, al punto de que no se interesa en la política: «Je me sentais aussi politisé qu'une serviette de toilette» (50) [me sentía tan politizado como un papel higiénico]. François es el académico petit-bourgeois de la globalización, centrado en sí mismo y sin conexión social, en tanto que desarrolla su existir en función de su cuerpo: «Mon corps qui ne pouvait plus être une source de plaisir» (205) [Mi cuerpo que no podía ser sino una fuente de placer].

François es definido fenomenológicamente con «el concepto de cuerpo vivido (Leib) (es decir, el cuerpo como este es experenciado por el ego personal)» ${ }^{30}$ (mi traducción); asume un uso fenomenológico del lenguaje; por ende, el lenguaje de la percepción ${ }^{31}$, lo cual permite interpretar su «yo tengo sexo», como «yo comprendo» ${ }^{32}$, en la medida que «tener sexo» es aprehender el mundo como cuerpo, sin mediación de la razón. Para la fenomenología, «el cuerpo es nuestro

29 Houellebecq, Soumission, 39; en adelante el número de página se indicará entre paréntesis en el texto. (Las traducciones entre corchetes son propios del autor del artículo. N. de la E.)

30 Sara Heinämaa, «The Body», The Routledge Companion to Phenomenology (Londres y New York: Routledge, 2012) 222.

31 Lewis R. Gordon, «Identity and Liberation: An Existential Phenomenological Approach», Phenomenology of the Political (Dordrecht: Kluwer Academic Publishers, 2000) 204.

32 Con respecto a esta idea, véase: Gordon, 204. 
modo familiar y primordial de habitar y mediar el "parlamento de las cosas", humanas y no-humanas ${ }^{33}$ (mi traducción), en tanto que hermenéutica carnal ${ }^{34}$, que implica «la privación de la participación en la vida comunal, el sentimiento de exilio y de desamparo ontológico» ${ }^{35}$ (mi traducción), lo cual es muy apto para los intereses de los grupos dominantes, en cuanto mecanismo de control social.

François es cuerpo que consume cuerpos, desprovisto de conciencia política y social. Este personaje es la estampa producida por el neoliberalismo fenomenologizante (con su lógica consumista post-postmoderna ${ }^{36}$ ), que sume al individuo en lo emocional (aspecto políticamente conservador) y le resta cualquier indicio de racionalidad (que podría ser profundamente contestataria). François vive en una torre de marfil, donde él y los demás académicos «se sentent absolument intouchables» (79) [se sienten absolutamente intocables], porque se sienten fuera del mundo, aunque reproduzcan la misma cotidianidad alienada y hedonista que aquellos que no lograron escalar con títulos universitarios, en la pirámide del consumismo hedonista-neoliberal que la globalización ha repartido, junto con la pobreza y la desigualdad social, por todo el mundo. François es menos que un mediocre, es un ideólogo. François cree ingenuamente que poseer es ser.

En su petite-bourgeoise burbuja, François no percibe lo económico-social, ya que él encarna la filosofía fenomenológica francesa desarrollada en, más o menos, los últimos sesenta años en Francia y que se ha corrido a todo el mundo, como movimiento ideológico reaccionario. La vivencia de François (su Erlebnis y su Ereignis) es una vivencia que se auto-representa sin sociabilidad, sin economía, sin

33 Hwa Yol Jung, «Carnal Hermeneutics and Political Theory», Political Phenomenology: Essays in Memory of Petee Jung (Suiza: Springer, 2016) 78. DOI: 10.1007/978-3-319-27775-2_6.

34 Jung, 81-82.

35 Bruno Viard, «Situation psycho-politique de Michel Houellebecq», Michel Houellebecq à la une (Amsterdam y New York: Rodopi, 2011) 134.

36 Jeffrey T. Nealon, Post-postmodernism or, the Cultural Logic of Just-in-time Capitalism (Stanford: Stanford University Press, 2012). 
materialidad; en donde François se percibe solo: «j'étais extrêmement seul» (73) [yo estaba extremamente solo].

François no tiene la realización personal que prometela educación de la era neoliberal (y que finalmente solo produce dóciles trabajadores $^{37}$ ); está reducido a una aislada pieza del engranaje de circulación/ reproducción del capital, semejante al esquema de la pedagogía del Banco Mundial, cuyo ideal de personalidad dentro de la economía del conocimiento, es un ser enfocado en sí mismo ${ }^{38}$. La existencia de François es solo lenguaje, una semiótica del (auto)consumismo, donde este (auto)consumismo se valora a sí mismo - en un proceso que freudianamente no pasa de ser una amalgama de mecanismos de defensa (racionalización, negación, formación reactiva, etc.) - como plenitud del Ser. Soumission, mediante la figura de François, nos permite acceder al cronotopo de la globalización, a la síntesis antropológica de un capitalismo en crisis terminal, o sea, al ser humano definido como decadencia, como soledad, como mediocridad, como animalidad irracional-emocional, como consumismo.

Sin embargo, todo el constructo lingüístico-semiótico que define al personaje tiene su antítesis, un elemento que rompe con la supuesta auto-poiesis de la complejidad del mundo artificioso de la intelligentsia petite-bourgeoise, a la que pertenece tal personaje. Ese mundo, ligado a una mágica complejidad, no escapa de a una realidad que se le filtra, al mundo de François, por hasta las ventanas. El elemento anti-tético, par excellence, es lo económico.

La economía condiciona en gran parte el funcionamiento del mundo auto-poiético, autogenerado, de François, sea como mundo del trabajo o, más específicamente, como salario. La inserción de François dentro del mundo del trabajo implica la pérdida de libertad: «Je venais de perdre quelque chose d'inappréciable, quelque chose que je ne retrouverais jamais : ma liberté» (15) [yo venía de perder algo inapreciable, algo que nunca reencontraría: mi libertad]. El

37 Michael W. Apple, Can Education Change Society? (Nueva York y Londres: Routledge, 2013) 4.

38 Joel Spring, Globalization of Education (Nueva York y Londres: Routledge, 2009) 43. 
mundo del trabajo conlleva, para François, el enfrentarse al espectro schopenhaueriano del sufrimiento (influencia de la que hablábamos arriba): «L'entrée dans la vie professionnelle plonge la plupart des êtres humaines dans une solitude aussi stupéfiante que radicale» (19) [el ingreso a la vida profesional sumerge a la mayor parte de los seres humanos en una soledad tan pasmada como radical]. El mundo del trabajo es experenciado, fenomenológicamente hablando, como una realidad extrema, producto de la alienación laboral del mundo globalizado y neoliberal, lo que sitúa la obra houellebecquiana «entre las raras ficciones francesas contemporáneas en abordar la cuestión de la representación de la sociedad consumista y de la alienación que en ella se desarrolla» ${ }^{39}$ (mi traducción).

Ante panorama tan sombrío, el hedonismo fenomenológiconeoliberal de François, no es más que un mecanismo de evasión. No obstante, este mecanismo mantiene a lo largo de Soumission una tensión dialéctica con lo económico, con la materialidad, a pesar de que François se ata irracionalmente a su «estilo de vida».

Lo económico se manifiesta en toda su relevancia, cuando a François lo obligan a pensionarse y en la medida que esto implica para él un descenso en su capacidad de consumo [que es finalmente lo que mantiene «viva» su fantasía petite-bourgeoise: «le montant mensual (...) s'élevait à ce jour à 3472 euros» (178) [el monto mensual (...) se elevaba en este momento a 3472 euros], lo cual desencadena una crisis:

J'étais dans la force de l'âge, aucune maladie létale ne me menaçait directement, les ennuis de santé qui m'assaillaient régulièreinent étaient douloureux mais somme toute mineurs ; ce n'est que dans une trentaine, voire une quarantaine d'années, que j'atteindrais cette zone sombre où les maladies deviennent toutes plus ou moins mortelles, où le pronostic vital, comme on dit, est presque à chaque fois engagé. Je n'avais pas d'amis, c'est certain, mais en avais-je jamais eu ? Et à quoi bon, si l'on voulait bien y réfléchir, des amis ? À partir

39 Matthieu Remy, «Michel Houellebecq et le décor de la société de consommation», Michel Houellebecq à la une (Amsterdam y New York: Radopi, 2011) 141. 
d'un certain niveau de dégradation physique - et cela irait beaucoup plus vite, il fallait compter une dizaine d'années, probablement moins, avant que la dégradation ne devienne visible, et qu'on ne me qualifie d'encore jeune - il n'y a plus qu'une relation de type conjugal qui puisse directement, et réellement, faire sens (183) [Yo estaba en la flor de la vida, ninguna enferma letal me amenazaba directamente, los problemas de salud que me atormentaban regularmente eran dolorosos pero de poca importancia; no son solo unos treinta, incluso unos cuarenta años, en que yo alcanzaría esta zona sombría donde las enfermedades devienen todas más o menos mortales, donde el pronóstico vital, como se dice, está casi cada vez comprometido. Yo no tenía amigos, es cierto, pero ¿los había yo tenido? Y ¿para qué, si se quiere reflexionar aquí, los amigos? A partir de un cierto nivel de degradación física -y esto iría mucho más de prisa, bastaría contar una decena de años, probablemente menos, antes de que la degradación solo devenga visible, y que uno ya no se califique como aún joven- no hay más que una relación de tipo conyugal que pueda directamente, y realmente, dar sentido.]

La realidad se cuela, finalmente, por la ventana de François, en cuanto lo económico rompe la visión del académico como algo intocable. El sistema hedonista-neoliberal y petit-bourgeois se fractura en su base. El discurso y la semiótica post-postmoderna del académico francés intocable se halla sin referencialidad; de hecho, el capitalismo «priva a todas las cosas y todo razonamiento de su conexión ontológica con la realidad ${ }^{40}$ (mi traducción), ante la materialidad del mundo y de su propio ser, la cual socaba su torre de marfil. Las inconsistencias del mundo de François se manifiestan aquí abiertamente, permitiendo captar que no había tal superioridad en ser parte de tal intelligentsia europea, su posición como trabajador se contrapone, en este proceso de degradación, a su auto-percepción identitaria, artificiosa y falsa de pequeño burgués universitario.

40 Andrea Micocci y Flavia di Mario, The Fascist Nature of Neoliberalism (Londres y New York: Routledge, 2018) 24. 
Houellebecq muestra un profundo conocimiento del desarrollo intelectual-filosófico francés. El escritor de Soumission contrapone, a través de la crisis de François, al desarrollo de la fenomenología francesa, post-estructuralista, el marxismo que tal fenomenología cercenó con su propio desarrollo políticamente reaccionario.

Dentro de un ambiente antimarxista (nos referimos al contexto de escritura, o sea, el 2015, año de publicación de Soumission), a partir de un juego temporal similar al extrañamiento cognitivo antes mencionado, Houellebecq propone una acción contestataria, que pone en duda el supuesto carácter absoluto de un neoliberalismo que se cree sin alternativa, a la vez que reafirma la preponderancia de lo económico, a través de la conversión al islam del propio François.

A François, contactado por Bastien Lacoue (quien trabaja para ediciones la Pléiade), lo eligen para hacer una edición de las obras de Huysmans, lo cual ve François como un golpe de suerte: «le fait est en tout case que je bénéficiais d'une espèce d'aura» (249) [el hecho es en todo caso que yo me beneficiaba de alguna especie de aura]. Tal «aura» permitirá a François recobrar no solo su capacidad de consumo, sino también su estatus social académico. No obstante, el proceso que inicia con la oferta de Lacoue a François, no es más que la expresión metonímica de un proceso más amplio, o sea, el hecho de que «les nations européennes n'étaient plus que des corps sans âme -des zombies» (255) [las naciones europeas no eran más que unos cuerpos sin alma -unos zombis].

Por otra parte, la decadencia de François-Europa muestra en Soumission tanto la fragilidad del académico, como lo prostituido y prostituible de la identitaria noción de europeidad. Como François sabe que «c'était vraiment la fin de ma vie intellectuelle» (283) [este era el fin de mi vida intelectual], también que «l'Europe était morte» (257) [Europa había muerta]. Es decir, el discurso (la semiótica) de la intelectualidad de François y el de la identidad de Europa se venden al mejor postor, lo cual afirma la irresponsabilidad para consigo mismos de François y de Europa. Al respecto, se lee en Soumission: «L'intellectuel 
en France n'avait pas à être responsable, ce n'était pas dans sa nature» (271) [el intelectual en Francia no tenía que ser responsable], con respecto a François, y «l'Europe occidentale n'était plus en état de se sauver elle-même» (276) [Europa Occidental no estaba más en capacidad de salvarse a sí misma], en relación con la Europa misma. También, la decadencia de François-Europa conlleva una afirmación de la preeminencia de lo económico sobre lo discursivo-semiótico.

Así, Houellebecq resuelve de golpe la tensión entre fenomenología y marxismo, superando (aufhebend) tal contradicción, mediante una ironía que no solo critica las nociones de intelectualidad y de europeidad hoy existentes, sino que también tal crítica implica en sí misma un fuerte ataque a la globalización, el neoliberalismo, la democracia burguesa, la petite bourgeoisie y la intelligentsia (francesa, europea y global). Robert Rediger se encarga de rédiger (redactar en francés) una nueva identidad para François y para Europa, proceso que demuestra la insubstancialidad del discurso que se aparta de la referencialidad y la hipocresía del Occidente primermundista, bajo la entropía de un capitalismo en quiebra.

\section{Houellebecq y la estética del realismo dialéctico}

En el momento actual en que circulan paradigmas anti-miméticos (anti-realistas) como las narrativas innaturales ${ }^{41}$ y la ciencia ficción del novum tecnocrático ${ }^{42}$, el retorno de Houellebecq al realismo - junto con el desplazamiento temporal y otras características de su obra - plantea una estética literaria acorde con nuestra posición como latinoamericanos, para no caer en la asunción de paradigmas poiético-narratológicos que responden a los intereses de los grupos

41 Roy Alfaro Vargas, «Las narrativas innaturales», Letras 60 (2016): 185-212. DOI: http://dx.doi. org/10.15359/rl.2-60.9.

42 Roy Alfaro Vargas, «El novum tecnocrático», Hélice II, 5 (2015): 6-20; Roy Alfaro Vargas, «The Protos Mandate: ciencia ficción y neoliberalismo», Letras 59 (2016): 77-97. DOI:http://dx.doi. org/10.15359/rl.1-59.4; Roy Alfaro Vargas, «Ciencia ficción cuántica y la ontología del conjunto vacío», Praxis 76 (2017): 91-106. DOI: http://dx.doi.org/10.15359/praxis.76.6. 
dominantes del Primer Mundo, y que no reflejan nuestra realidad, como proveedores de mano de obra barata y de materias primas.

En contraposición con las narrativas innaturales y el novum tecnocrático, adaptamos la idea del realismo dialéctico. Este realismo responde a una estética cognitiva, en tanto la literatura (y toda forma de arte) es un proceso de aprehensión de la realidad, lo que quiere decir que la literatura es mimética y referencial. Así, el fenómeno literario, en particular, y los demás productos culturales estéticos (cine, pintura, etc.), en general, son un proceso racional, que en cuanto tales se anclan en lo real.

Los productos culturales estéticos, en tanto reales y racionales, son formas de discontinuidad, que articulan en términos lingüísticosemióticos la continuidad, y, dentro de esta perspectiva, estos sirven para aprehender críticamente lo real, en función de lo posible. O sea, la diégesis literaria, fílmica, etc., siempre refiere a factores extradiegéticos, ónticamente presentes o como posibles desarrollos ontológicos ligados a una praxis social.

El desplazamiento temporal mencionado, con respecto a Soumission, si bien por un lado permite distanciar al lector/espectador de su contexto, con el fin de representarle su realidad (y algunas posibles soluciones a las problemáticas existentes en ella) sin la carga emocional que implica para él/ella indudablemente tal contexto; lo cierto es que igualmente y en términos ontológicos es posible acceder al tiempo total, o sea, al tiempo como articulación de pasado, presente y futuro; como flecha del tiempo, lo cual en este caso permite observar el capitalismo en su proceso entrópico, en su degradación y decadencia, lo cual evita substancializarlo y, a la vez, creerlo eterno al estilo de un sistema complejo auto-organizado ${ }^{43}$; es decir, como un sistema capaz de reordenarse, sin mediar condición previa alguna, para alcanzar su

43 Actualmente, algunos grupos académicos reaccionarios de ultra-derecha pretenden que el capitalismo no es un «modo de producción», sino alguna clase de complejo social e institucional, es decir, un sistema complejo que se auto-organiza. Al respecto, véase: Stephen Maher y Scott M. Aquanno, «Conceptualizing Neoliberalism: Foundations for an Institutional Marxist Theory of Capitalism», New Political Science 40, 1 (2018): 33-50. DOI:10.1080/07393148.2017.141672. En 
objetivo $^{44}$. El tiempo deviene en esta estética dialéctico-realista, la posibilidad del Ser, pero también en la superación(Aufhebung) de este, en cuanto Deber Ser. Es decir, se asume lo existente en función de un posible proyecto político, que rompa con los procesos de exclusión, explotación, etc., que derivan del modo de producción capitalista.

La crítica houellebecquiana al neoliberalismo y a la globalización, se asume en esta estética realisto-dialéctica como una política de lo posible, con lo cual es viable una crítica del neoliberalismo (entendido en términos políticos, económicos y civiles), en tanto este responde a una estrategia de la burguesía para salir de la crisis sistémica, que corroe al capitalismo desde la década de 1970 y donde la crisis del 2007-8 permitió visualizar la inviabilidad del proyecto neoliberal y del capitalismo ${ }^{45}$.

En este marco, el realismo dialéctico se opone a la política de lo imposible, la cual se caracteriza no solo por su carácter reaccionario, como estrategia de control social y político, sino también por su énfasis anti-mimético y arreferencial ${ }^{46}$. Así, el realismo dialéctico, en contraposición a la política de lo imposible, se define en términos sociológicos y políticos, como un enfoque contestatario y, de hecho, anti-neocolonialista, con respecto a paradigmas como las narrativas innaturales y el novum tecnocrático.

El realismo dialéctico retoma la descriptibilidad y la objetividad del realismo decimonónico, pero, mediante el juego temporal (tomado de la ciencia ficción), no se describe una realidad estática centrada en el principio de identidad $(\mathrm{A}=\mathrm{A})$ de la lógica formal; la descripción se asume como congruencia $(\mathrm{A} \equiv \mathrm{A}$, o sea, se articula lo real y lo posible) para aprehender-construir la realidad como movimiento,

este marco, desde de nuestra perspectiva, la posición de Maher y Aquanno implica que el capitalismo sería eterno, lo cual es falso por el principio de entropía mismo.

44 Raúl Sánchez y David Newman, «Primer on Complex Systems», A Primer on Complex Systems (Dordrecht: Springer, 2018) 13. DOI:https://doi.org/10.1007/978-94-024-1229-1_1.

45 Chris Harman, Zombie Capitalism. Global Crisis and the Relevance of Marx (Londres: Bookmarks, 2009).

46 Alfaro Vargas, «Las narrativas innaturales», 209-212. 
como cambio, como transformación social. Además, la objetividad se supera (aufhebt) dialécticamente, en cuanto el escritor, el director, etc., parten de una posición política crítica del capitalismo, la globalización y el neoliberalismo. Es decir, es una objetividad que recupera el sujeto como agente histórico, retomando la unidad gnoseológica de sujeto y objeto.

Fundamental es el empleo filosófico, como lo ha mostrado Houellebecq. Un profundo enfoqueo filosófico les permite al escritor, al director, etc., no solo comprender las sutilezas del discurso político que hoy se reviste de fenomenología, sino también presentar estéticamente estrategias para denunciar las incongruencias y mentiras ideológicas que el capitalismo genera para sobrevivir, así como plantear estrategias contestatarias contra el sistema capitalista.

Comprender el énfasis en lo emocional que el neoliberalismo fenomenologizante imprime hoy a la cultura es clave para enfrentarlo. El realismo dialéctico es, por ende, tanto un retorno a la razón como un poner en su justo lugar a la emoción.

\section{Conclusiones}

Houellebecq, en Soumission, establece una dialéctica entre fenomenología y materialidad, apuntando a la cuestión del hedonismo y de la economía, respectivamente, como un medio de crítica política y anti-académica, a la vez que deja implícitos los fundamentos para una estética del realismo dialéctico.

Al centrarse en François, Soumission representa a la intelligentsia francesa como mediocre, sin compromiso social y sin racionalidad alguna, lo cual nos hace pensar cuál sería la condición de aquellos sectores de la sociedad que no han tenido acceso a la educación (universitaria o mucho menos), si los académicos de esta sociedad houellebecquiana presentan un nivel tan bajo y son incluso capaces de venderse al mejor postor. 
Fiel a su estilo, mediante la ironía, la abierta burla, su naturalismo de corte social y biologicista ${ }^{47}$, su materialismo, etc., Houellebecq muestra una Francia, una Europa y un capitalismo globalizante en su carácter alienante, que no permite el desarrollo pleno del ser humano; sino que sume al ser humano en su instintividad, en su forma más primaria, en su animalidad hedonista cuyo placer no es más que el mecanismo de control social de una burguesía que ve pronta su extinción.

El desplazamiento temporal que ejecuta Houellebecq (junto con su crítica política), al modo del extrañamiento cognitivo de la ciencia ficción, plantea algunos elementos para desarrollar la estética del realismo dialéctico, en contraposición con los proyectos ideológico-poéticos y narratológicos de las narrativas innaturales y del novum tecnocrático de la ciencia ficción. Así, el realismo dialéctico se plantea como opción estética, acorde con el contexto de nuestros países latinoamericanos ante planteamientos neocolonialistas, como las ya mencionadas narrativas innaturales. Queda por desarrollar más a profundidad la noción de realismo dialéctico, que aquí queda tan solo esbozada.

47 Rabosseau, 43. 
